\title{
The ASY-EOS experiment at GSI: investigating symmetry energy at supra-saturation densities
}

P. Russotto1,a, M. Chartier², M.D. Cozma ${ }^{3}$, E. De Filippo ${ }^{1}$, A. Le Fèvre ${ }^{4}$, S. Gannon², I. Gašparić5,6, M. Kiš ${ }^{4,5}$, S. Kupny ${ }^{7}$, Y. Leifels ${ }^{4}$, R.C. Lemmon ${ }^{8}$, Q. Li ${ }^{9}$, J. Łukasik $^{10}$, P. Marini ${ }^{11,12}$, P. Pawłowski ${ }^{10}$, S. Santoro ${ }^{13,14}$, W. Trautmann ${ }^{4}$, M. Veselsky ${ }^{15}$, L. Acosta ${ }^{16}$, M. Adamczyk ${ }^{7}$, A. Al-Ajlan ${ }^{17}$, M. Al-Garawi ${ }^{18}$, S. Al-Homaidhi ${ }^{17}$, F. Amorini ${ }^{16}$, L. Auditore ${ }^{13,14}$, T. Aumann ${ }^{6}$, Y. Ayyad $^{19}$, V. Baran ${ }^{16,20}$, Z. Basrak ${ }^{5}$, R. Bassini21 ${ }^{21}$ J. Benlliure ${ }^{19}$, C. Boiano ${ }^{21}$, M. Boisjoli ${ }^{12}$, K. Boretzky ${ }^{4}$, J. Brzychczyk ${ }^{7}$, A. Budzanowski ${ }^{10}$, G. Cardella ${ }^{1}$, P. Cammarata ${ }^{11}$, Z. Chajecki22, A. Chbihi' ${ }^{12}$, M. Colonna ${ }^{16}$, B. Czech ${ }^{10}$, M. Di Toro ${ }^{16,23}$, M. Famiano ${ }^{24}$, V. Greco ${ }^{16,23}$, L. Grassi' ${ }^{5}$, C. Guazzoni ${ }^{21,25}$, P. Guazzoni ${ }^{21,26}$, M. Heil ${ }^{4}$, L. Heilborn ${ }^{11}$, R. Introzzi ${ }^{27}$, T. Isobe ${ }^{28}$, K. Kezzar ${ }^{18}$, A. Krasznahorkay ${ }^{29}$, N. Kurz ${ }^{4}$, E. La Guidara ${ }^{1}$, G. Lanzalone ${ }^{16,30}$, P. Lasko ${ }^{7}$, I. Lombardo ${ }^{31,32}$, W.G. Lynch ${ }^{22}$, Z. Matthews ${ }^{3}$, L. May ${ }^{11}$, T. Minniti ${ }^{13,14}$, M. Mostazo ${ }^{19}$, A. Pagano ${ }^{1}$, M. Papa ${ }^{1}$, S. Pirrone ${ }^{1}$, R. Pleskac ${ }^{4}$, G. Politi ${ }^{1,23}$, F. Porto ${ }^{16,23}$, R. Reifarth ${ }^{4}$, W. Reisdorf ${ }^{4}$, F. Riccio ${ }^{21,25}$, F. Rizzo ${ }^{16,23}$, E. Rosato ${ }^{31,32}$, D. Rossi ${ }^{4,22}$, H. Simon ${ }^{4}$, I. Skwirczynska ${ }^{10}$, Z. Sosin ${ }^{7}$, L. Stuhl29,

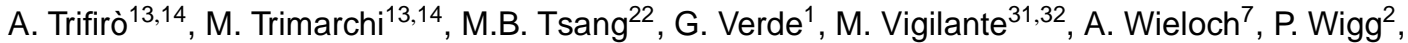
H.H. Wolter ${ }^{33}$, P. Wu ${ }^{2}$, S. Yennello ${ }^{11}$, P. Zambon ${ }^{21,25}$, L. Zetta ${ }^{21,26}$, and M. Zoric ${ }^{5}$

${ }^{1}$ INFN-Sezione di Catania, Catania, Italy

${ }^{2}$ University of Liverpool, Liverpool, UK

${ }^{3}$ IFIN-HH, Magurele-Bucharest, Romania

${ }^{4}$ GSI Helmholtzzentrum, Darmstadt, Germany

${ }^{5}$ Ruder Boškovic̀ Institute, Zagreb, Croatia

${ }^{6}$ Technische Universität, Darmstadt, Germany

${ }^{7}$ Jagiellonian University, Krakòw, Poland

${ }^{8}$ STFC Laboratory, Daresbury, UK

${ }^{9}$ Huzhou Teachers College, China

${ }^{10}$ IFJ-PAN, Krakow, Poland

${ }^{11}$ Texas A\&M University, College Station, USA

${ }^{12}$ GANIL, Caen, France

${ }^{13}$ INFN-Gruppo Collegato di Messina, Messina, Italy

${ }^{14}$ Università di Messina, Messina, Italy

${ }^{15}$ Institute of Physics, Slovak Academy of Sciences, Bratislava, Slovakia

${ }^{16}$ INFN-Laboratori Nazionali del Sud, Catania, Italy

${ }^{17}$ KACST Riyadh, Riyadh, Saudi Arabia

${ }^{18}$ King Saud University, Riyadh, Saudi Arabia

${ }^{19}$ University of Santiago de Compostela, Santiago de Compostela, Spain

${ }^{20}$ University of Bucharest, Bucharest, Romania

${ }^{21}$ INFN-Sezione di Milano, Milano, Italy

${ }^{22}$ NSCL Michigan State University, East Lansing, USA

${ }^{23}$ Università di Catania, Catania, Italy

\footnotetext{
${ }^{\mathrm{a}} \mathrm{e}$-mail: russotto@1ns.infn.it
} 


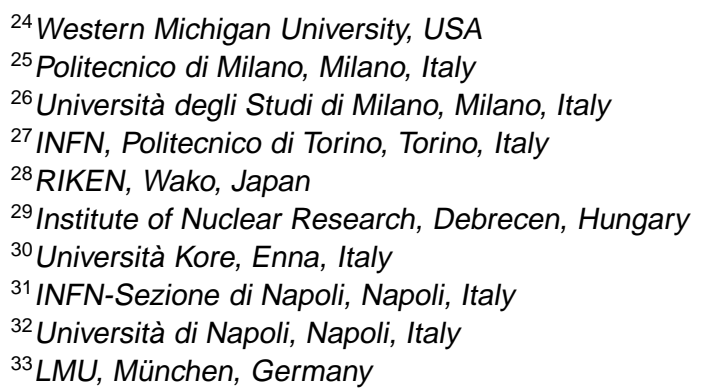

\begin{abstract}
The elliptic-flow ratio of neutrons with respect to protons or light complex particles in reactions of heavy-ions at pre-relativistic energies has been proposed as an observable sensitive to the strength of the symmetry term of the nuclear equation of state at supra-saturation densities. The results obtained from the existing FOPI/LAND data for ${ }^{197} \mathrm{Au}+{ }^{197} \mathrm{Au}$ collisions at $400 \mathrm{MeV} /$ nucleon in comparison with the UrQMD model simulations favoured a moderately soft symmetry term, but suffer from a considerable statistical uncertainty. These results have been confirmed by an independent analysis based on the Tübingen QMD simulations. In order to obtain an improved data set for $\mathrm{Au}+\mathrm{Au}$ collisions and to extend the study to other systems, a new experiment was carried out at the GSI laboratory by the ASY-EOS collaboration. The present status of the data analysis is reported
\end{abstract}

\title{
1 ASY-EOS (S394) experiment at GSI
}

The neutron-proton elliptic flows ratio and difference have been proposed as useful observables in order to investigate the high-density behavior of the nuclear symmetry energy [1,2]. Comparisons of existing data from the FOPI-LAND experiment [3] with UrQMD and Tübingen QMD model calculations suggest a moderately soft symmetry term, but suffer from a considerable statistical uncertainty. The agreement of constraints obtained with a momentum-independent isovector model (UrQMD) and with a momentum-dependent one (Tübingen QMD ) suggests a route towards a model independent constraint of the the high-density symmetry energy [4]. In order to improve the statistical accuracy of measured flow parameters for $\mathrm{Au}+\mathrm{Au}$ reactions and to extend the flow measurements to other systems, the symmetric collision systems ${ }^{197} \mathrm{Au}+{ }^{197} \mathrm{Au},{ }^{96} \mathrm{Zr}+{ }^{96} \mathrm{Zr}$ and ${ }^{96} \mathrm{Ru}+{ }^{96} \mathrm{Ru}$ at $400 \mathrm{MeV} /$ nucleon incident energies have been measured in May 2011 at GSI (S394 exp.). In this new experiment, the study of isospin effects is extended to additional observables as, e.g., the yield ratios of light isobars pairs ${ }^{3} \mathrm{H} /{ }^{3} \mathrm{He}$ and ${ }^{7} \mathrm{Li} /{ }^{7} \mathrm{Be}$.

A schematic view of the experimental set-up is shown in Fig. 1. The Large Area Neutron Detector (LAND) [5] was positioned at laboratory angles around $45^{\circ}$ with respect to the beam direction, at a distance of about $5 \mathrm{~m}$ from the target. A veto-wall of plastic scintillators in front of LAND allowed discrimination of neutrons and charged particles. In this configuration it was possible to measure the direct and elliptic flows of neutrons and hydrogens at mid-rapidity with high precision in the same angular acceptance. In addition, the Krakòw Triple Telescope Array, KraTTA [6], was installed to measure the energy, emission angles and isotopic composition of light charged reaction products. The 35 modules of KraTTA, arranged in a 7x5 array, were placed opposite to LAND at the distance of 40 $\mathrm{cm}$ from the target, and covering $160 \mathrm{msr}$ of the solid angle, at polar angles between $20^{\circ}$ and $64^{\circ}$. The modules of KraTTA consisted of two, optically decoupled, CsI(Tl) crystals (thickness of 2.5 and 12.5 $\mathrm{cm}$ ) and three large area, $500 \mu \mathrm{m}$ thick, PIN photo-diodes. Very good isotopic resolution has been 
obtained in the whole dynamic range (see [6]) up to $\mathrm{Z} \sim 6$.

The determination of the impact parameter and the orientation of the reaction plane required the use of several devices: i) the ALADIN Time-of-Flight (AToF) wall [7] was used to detect forward emitted charged particles at polar angles smaller than $7^{\circ}$; two walls (front and rear) of $2.5^{*} 100 \mathrm{~cm}^{2}$ plastic scintillators, read by two photo-multipliers at both ends, gave information on emission angle, atomic number and velocity of ions; ii) 50 thin (between 3.6 and $5.6 \mathrm{~mm}$ ) CsI(Tl) elements, read out by photo-diodes, arranged in 4 rings of the Washington-University $\mu$ Ball array [8], covering polar angles between $60^{\circ}$ and $147^{\circ}$, surrounded the target with the aim of measuring the distribution of backward emitted particles and to discriminate against background reactions on non-target material; iii) $352 \mathrm{CsI}(\mathrm{Tl})$ scintillators, $12 \mathrm{~cm}$ thick, of the CHIMERA multidetector [9], arranged in 8 rings in $2 \pi$ azimuthal coverage around the beam axis, covering polar angles between $7^{\circ}$ and $20^{\circ}$, measured the emission of light charged particles.

With beam intensities of about $10^{5} \mathrm{pps}$ and targets of $1-2 \%$ interaction probability, about $5 * 10^{6}$ events for each system were collected. Special runs were performed with and without target, in order to measure the background from interaction of projectile ions with air, and with iron Shadow Bars covering the angular acceptance of LAND in order to measure neutron background. The analysis of the collected data is currently in progress. As a global result, we show in Fig. 2 the invariant plot of transverse velocity vs. rapidity in the laboratory reference system for charged particles detected by the AToF, CHIMERA and $\mu$ Ball and for neutrons registered by the first plane of LAND detector for a sample of the $\mathrm{Au}+\mathrm{Au}$ data. The plot is not corrected for detection efficiency; in the case of $\mu \mathrm{Ball}$, since an energy calibration is at the moment not available, we have adopted a uniform kinetic energy distribution between 0 and $100 \mathrm{MeV}$. We can clearly see the population of the intense regions at midrapidity and around projectile rapidity; since the AToF has a reduced $(\sim 50 \%)$ efficiency in detecting Hydrogens, a jump in the yield is seen in the matching region between AToF and CHIMERA $(\sim 100$ $\%$ efficiency for the detection of Light Charged Particles). In the whole kinematic range between CHIMERA and $\mu$ Ball, isotopic identification of charged particles was obtained in the KraTTA data (not included in this plot). Selection of reaction centrality and background rejection can be performed by looking at the correlations between global variables as extracted from the data of a given subdetector.

An important feature of the flow measurement is the resolution achieved in determining the azimuthal orientation of the reaction plane because it largely determines the uncertainty associated with the determined flow parameters [10]. As a first test we have estimated the reaction plane orientation from CHIMERA, by using the Q-vector method of Ref. [10]. By applying the sub-event mixing technique of [11], a resolution on reaction plane reconstruction of $\Delta \phi<\sim 30^{\circ}$ was obtained, quite satisfactory and in agreement with the accuracy expected in the simulations. Finally Fig. 3 shows the azimuthal

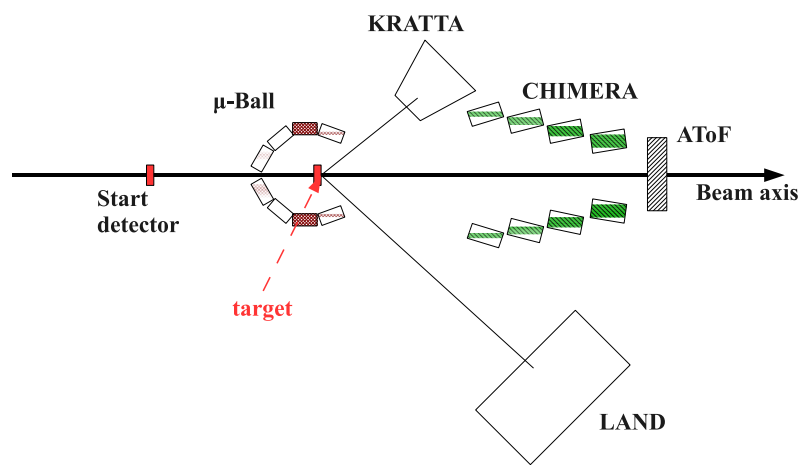

Figure 1. Schematic view, not to scale, of the experimental setup of the ASY-EOS (S394) experiment at GSI 


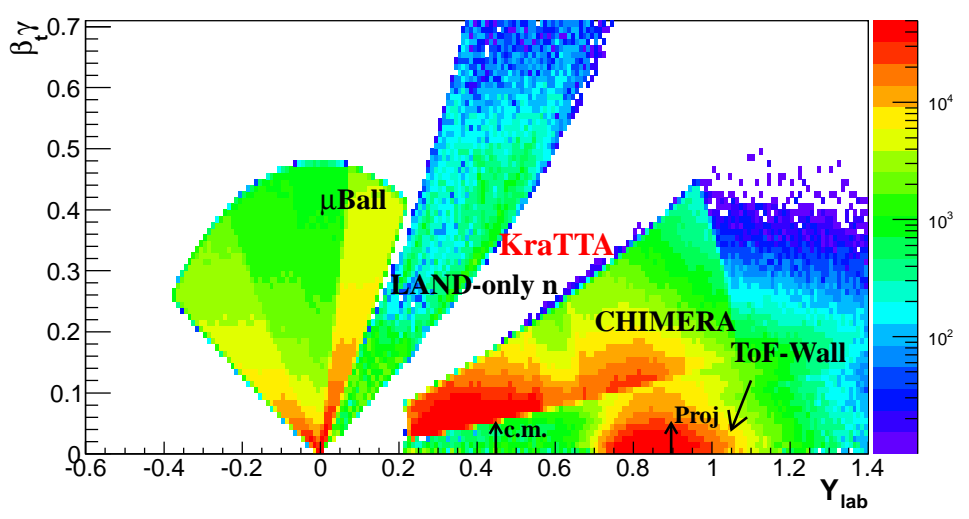

Figure 2. Transverse velocity versus rapidity in laboratory reference system for charged particles detected by AToF, CHIMERA and $\mu$-Ball array and for neutrons detected by LAND for $\mathrm{Au}+\mathrm{Au}$ data at 400 $\mathrm{MeV} /$ nucleon.

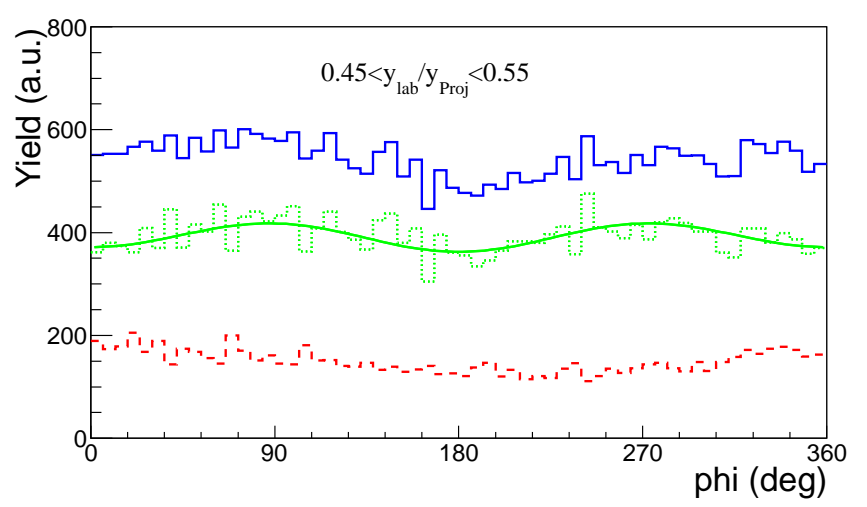

Figure 3. Azimuthal distributions of neutrons for $\mathrm{Au}+\mathrm{Au}$ collisions measured without (blue continuous line) and with shadow bar (dashed red line). Fitting the difference (green dotted line) with Eq. (1) of [1] yields $v_{2}=-0.10 \pm 0.01$, after correcting for reaction plane dispersion.

distribution of neutrons around mid-rapidity, extracted from the $1^{\text {st }}$ plane of LAND, with respect to the reaction plane determined by CHIMERA, for a selected sample of the Au+Au data with impact parameter $b<7.5 \mathrm{fm}$. The expected squeeze-out is clearly seen. Once data reduction will be completed, the ASY-EOS experiment is expected to provide a data set that will allow careful tests of reaction models and the ability to put constraints on the symmetry energy [4].

\section{References}

[1] P. Russotto et al., Phys. Lett. B 697, 471 (2011)

[2] M.D. Cozma, Phys. Lett. B 700, 139 (2011)

[3] Y. Leifels et al., Phys. Rev. Lett. 71, 963 (1993)

[4] M.D. Cozma et al., arXiv:1305.5417 [nucl-th] (2013), submitted to Phys. Rev. C

[5] Th. Blaich et al., Nucl. Instr. and Meth. A 314, 136 (1992)

[6] J. Łukasik et al., Nucl. Instr. and Meth. A 709, 120 (2013)

[7] A. Schüttauf et al., Nucl. Phys. A 607, 457 (1996)

[8] D.G. Sarantites et al., Nucl. Instr. and Meth. A 381, 418 (1996)

[9] A. Pagano et al., Nucl. Phys. A 734, 504 (2004)

[10] A. Andronic et al., Eur. Phys. J. A 30, 31 (2006)

[11] J.Y. Ollitrault, arxiv:nucl-ex/9711003 (1997) 